\title{
A Coordinate Axis Transformation Study of Spatial QRS Loop in Hypertrophic Cardiomyopathy
}

\author{
Akihiko TakimiYa, Shuichi NaKaJima, Motoyuki MugikURA, Kenichi MutOH and \\ Chiharu IBUKIYAMA
}

\begin{abstract}
To obtain an overall view of the QRS loop on vectorcardiograms (VCG) of hypertrophic cardiomyopathy (HCM), the coordinate axis was transformed using the resolver method. The morphological features and planarity of the loop were compared with hypertrophic patterns and hypertensive heart disease (HHD). The subjects in the present study included 30 normal individuals, 40 patients with HCM and 30 with HHD. The HHD group was selected from patients showing left ventricular hypertrophy on VCG similar to that of HCM patients. The HCM group showed significantly greater values than the HHD group in the thickness/length ratio, which represents the planarity of the spatial QRS loop. The above finding suggests that the HCM group had greater deformation in the QRS loop than the HHD group. This may provide a useful indicator for the differential diagnosis of the two diseases.
\end{abstract}

Key words: Planarity, Broadside view, Edgewise view, Resolver

Hypertrophic cardiomyopathy (HCM) is known to show various patterns on standard 12-lead electrocardiograms $(1-5)$. It has also been reported that the disease presents left ventricular hypertrophy or an early defect of the septal vector on vectorcardiograms (VCG) (6). Most of the above findings, however, are not necessarily specific to HCM, and sometimes appear on VCG of hypertensive heart disease (HHD), and therefore there is a need to differentiate between HCM and HHD in clinical practice.

In the present study, we obtained an overall view of the spatial QRS loop from conventional VCG by using the resolver method (7) rather than the general measurements. The morphological features, planarity in particular, of the spatial loop were studied in comparison with hypertrophic patterns in HCM patients. Differences in these factors were also examined in HHD patients showing left ventricular hypertrophy similar to HCM on VCG.

\section{MATERIALS AND METHODS}

The present study included 100 subjects: 30 normal individuals $(\mathrm{N})$ (19 males and 11 females; mean age 56.2 \pm 13.3 ), 30 HHD patients ( 21 males and 9 females; mean age $54.1 \pm 11.8$ ) and $40 \mathrm{HCM}$ patients (29 males and 11 females; mean age $48.3 \pm 13.1)$. The HCM group was further divided according to hypertrophic patterns on M-mode echocardiograms into 14 patients with asymmetrical septal hypertrophy (ASH), 12 with apical hypertrophy (APH) and 14 with diffuse hypertrophy (DH). Patients with hypertrophic obstructive cardiomyopathy (HOCM) were excluded from the study (Fig. 1).

We investigated the patients by echocardiography; in particular, the apical view was examined in detail. To determine the APH group, we screened those patients who indicated all of the following characteristics: was localized hypertrophy in the

From Second Department of Internal Medicine, Tokyo Medical College Hospital, Tokyo

Received for publication May 5, 1990; Accepted for publication September 19, 1990

Reprint requests should be addressed to Akihiko Takimiya, MD, Second Department of Internal Medicine,

Tokyo Medical College Hospital, 7-1 Nishishinjuku 6-chome, Shinjuku-ku, Tokyo 160, Japan 


\begin{tabular}{c|c|c}
\hline & IVST & LVPWT \\
\hline ASH & $>15 \mathrm{~mm}$ & $\leqq 12 \mathrm{~mm}$ \\
\hline APH & $\leqq 15 \mathrm{~mm}$ & $\leqq 12 \mathrm{~mm}$ \\
\hline DH & $>15 \mathrm{~mm}$ & $>12 \mathrm{~mm}$ \\
\hline
\end{tabular}

ASH : Asymmetric septal hypertrophy type

APH : Apical hypertrophy type

$\mathrm{DH}$ : Diffuse hypertrophy type

IVST : Interventricular septum thickness

LVPWT : Left ventricular posterior wall thickness

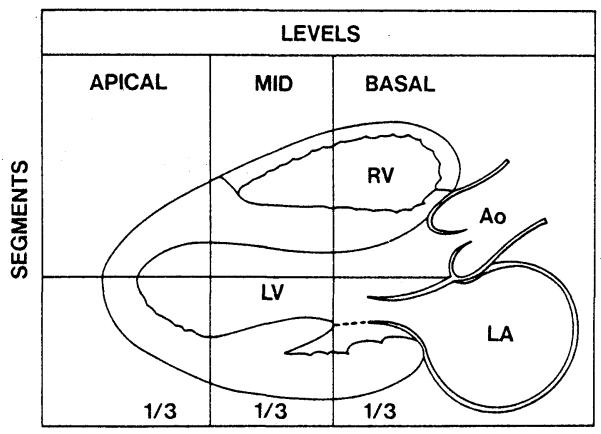

Fig. 1. The HCM group was divided into 3 groups according to the hypertrophic patterns on M-mode echocardiograms.

region of apex cordis; on LVG, a spade shape appeared at systole; and further findings specific to HCM identified by biopsy samples. We believe the above test results are essential as well as sufficient to diagnose APH. To select patients included in the DH group, we confirmed that the patient was not hypertensive by careful examination of the detailed medical history. Further, confirmation of HCM was made by biopsy samples. The HOCM group consisted of those patients whose hypertrophy of the septum and SAM of the mitral valve were recognized by echocardiography, and further by the cardiac catheterization test, a pressure gradient was recognized on the pull-back pressure from left ventricular to aorta. In HOCM cases, we thought it is necessary to exclude the effect on the ventricular muscle accompanying the unique hemodynamic change at the left ventricle.

The HHD patients selected for study were those with a systolic blood pressure of higher than $160 \mathrm{~mm}$ $\mathrm{Hg}$ and a diastolic pressure of higher than $95 \mathrm{~mm}$ $\mathrm{Hg}$ on at least three previous blood pressure measurements, and those who met all of the following VCG criteria for left ventricular hypertrophy.

1) The magnitude of the maximum vector in the horizontal plane was increased more than 2.0 $\mathrm{mV}$ and showed left posterior displacement.

2) The ST vector was directed significantly to the right.

3) The QRS-T angle was widened.

HHD patients thus selected showed no significant differences from the HCM group in terms of the 1) magnitude and angle of the maximal vector of the QRS loop in horizontal and frontal planes, 2) magnitude and arrival time to the maximal vector of the spatial QRS loop, 3) duration of QRS loop, and 4) magnitude in the component scaler X-lead. In order to investigate the variations of QRS due to histopathological differences, the HHD group was made up of selected cases that indicated no significant differences with the HCM group on the VCG. Thus, there was no specific limitation as to the type and degree of cardiac hypertrophy on the echocardiogram. There was no significant difference in parameters on the ECG between those groups.

Patients with apparent conduction disturbance such as bundle-branch block or those with the complication of ischemic heart disease were also excluded. Three techniques were used to exclude ischemic heart diseases: confirmation of no mural exercise abnormality on the echocardiogram, no decline or deficiency of Tl-Cl uptake, and no significant coronary artery lesion by cardiac catheterization.

Scalar signals at $\mathrm{X}, \mathrm{Y}$ and $\mathrm{Z}$ in the Frank lead system were obtained from all subjects using a VA-3H vectorcardiograph (Fukuda Denshi Co., Ltd., Tokyo, Japan). Employing the method of Schmitt (8) and Milnor et al (9), the signals were transformed to obtain the maximal loop (broadside view) on the Braun tube location equivalent to the horizontal plane using a $3-\mathrm{knob}$ coordinate axis transformer set in the vectorcardiograph. Coordinates $\mathrm{X}, \mathrm{Y}$ and $\mathrm{Z}$ were transformed by this manipulation into the coordinates $\mathrm{X}^{\prime \prime}{ }^{\prime}, \mathrm{Y}^{\prime \prime \prime}$ and $\mathrm{Z}^{\prime \prime}$ ' by the following three formulas:
1) $\left[\begin{array}{l}\mathrm{X}^{\prime}=\mathrm{X} \cos \mathrm{A}-\mathrm{Z} \sin \mathrm{A} \\ \mathrm{Y}^{\prime}=\mathrm{Y} \\ \mathrm{Z}^{\prime}=\mathrm{Z} \cos \mathrm{A}+\mathrm{X} \sin \mathrm{A}\end{array}\right.$
...(rotation $\mathrm{A})$
2) $\left[\begin{array}{l}\mathrm{X}^{\prime \prime}=\mathrm{X}^{\prime} \\ \mathrm{Y}^{\prime \prime}=\mathrm{Y}^{\prime} \cos \mathrm{B}-\mathrm{Z}^{\prime} \sin \mathrm{B} \\ \mathrm{Z}^{\prime \prime}=\mathrm{Z}^{\prime} \cos \mathrm{B}+\mathrm{Y}^{\prime} \sin \mathrm{B}\end{array}\right.$
...(rotation $\mathrm{B})$ 
3) $\left[\begin{array}{l}\mathrm{X}^{\prime \prime \prime}=\mathrm{X}^{\prime \prime} \cos \mathrm{C}-\mathrm{Y}^{\prime \prime} \sin \mathrm{C} \\ \left.\mathrm{Y}^{\prime \prime \prime}=\mathrm{Y}^{\prime \prime} \cos \mathrm{C}+\mathrm{X}^{\prime \prime} \sin \mathrm{C} \quad \text {...(rotation } \mathrm{C}\right) \\ \mathrm{Z}^{\prime \prime \prime}=\mathrm{Z}^{\prime \prime}\end{array}\right.$

At this point, the view equivalent to the left sagittal plane is the edgewise view of the spatial loop. The length (a), width (b) and area of the spatial QRS loop were calculated from the broadside view obtained by the above manipulation, and thickness (c) from the edgewise view (Fig. 2). At the same time, the $\mathrm{c} / \mathrm{a}$ and $\mathrm{a} / \mathrm{b}$ ratios were calculated. These factors were then compared between groups.

\section{RESULTS}

The area of the spatial QRS loop in the HHD group and each HCM subgroup was significantly larger than that in the normal group $(p<0.01)$. There was no significant difference between disease groups (Fig. 3). The thickness (c) of the spatial QRS loop was significantly greater in the HHD and HCM groups than in the normal group $(p<0.01)$. The APH group showed significantly greater values than the ASH and HHD groups ( $<<0.1, \mathrm{p}<0.05$ ) (Fig. 4).

The c/a ratio was significantly greater in the HCM group than in the other two groups $(\mathrm{p}<0.05)$. No significant difference was found between the

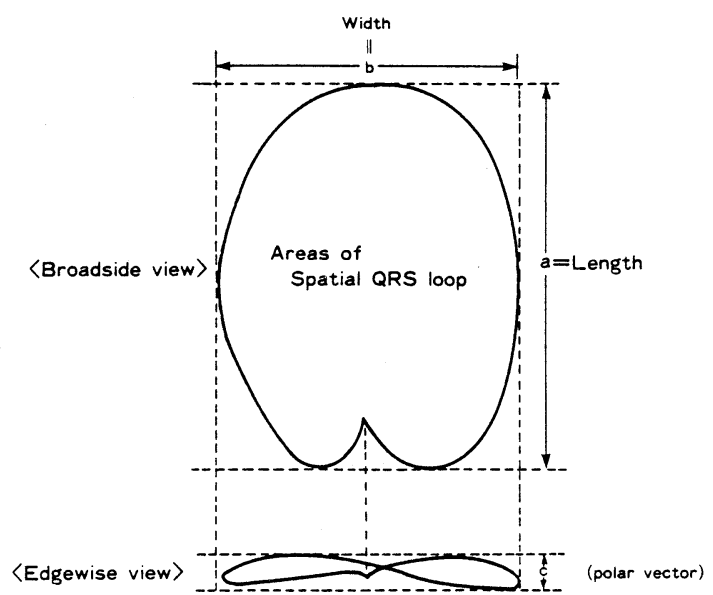

Fig. 2. Schematic representation of the measurements taken using broadside and edgewise views of spatial QRS loops. Length (a) was measured through the two most distant points of the QRS loop. Width (b) was also measured through the most distant points, perpendicular to (a). The maximal transverse diameter of QRS loop in "edgewise view" is indicated by thickness (c). The c/a ratio was used in the analysis as an indicator of planarity of the QRS loop.
HHD group and the normal group (Fig. 5). All the HCM subgroups showed a significantly greater increase in the ratio than the HHD group $(\mathrm{p}<0.01$, $\mathrm{p}<0.1$ ) or a tendency toward increase. There was no significant difference between HCM subgroups (Fig. 6). The $\mathrm{a} / \mathrm{b}$ ratio was not significantly different between groups (Fig. 7).

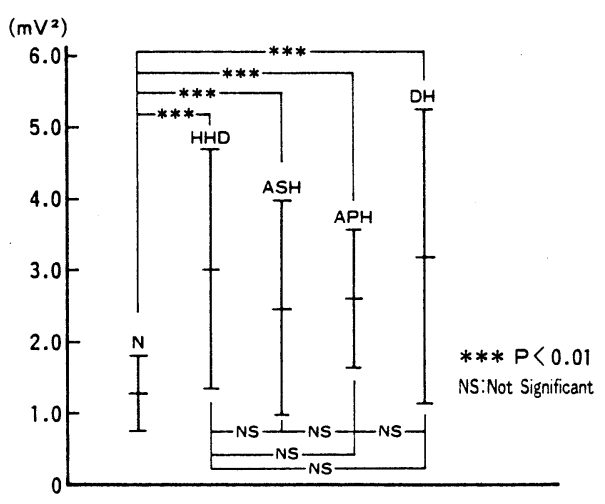

Fig. 3. Bar graph illustrating the area of the spatial QRS loop. There is no significant difference between the HHD group and each HCM subgroup. APH, apical hypertrophy; ASH, assymmetrical septal hypertrophy; DH, diffuse hypertrophy; HHD, hypertensive heart disease; N, normal group; NS, not significant

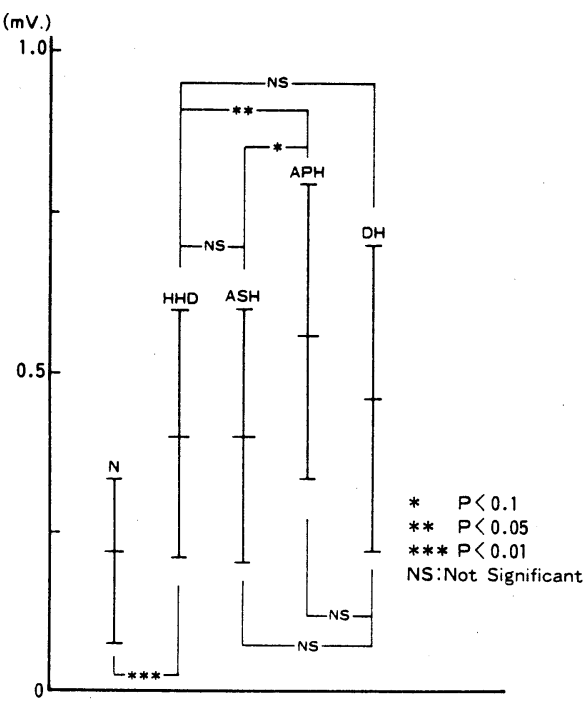

Fig. 4. Bar graph illustrating the thickness (c) of the spatial QRS loop. The thickness is significantly greater in the HHD and HCM groups than in the normal group. Abbreviations are the same as Fig. 3. 


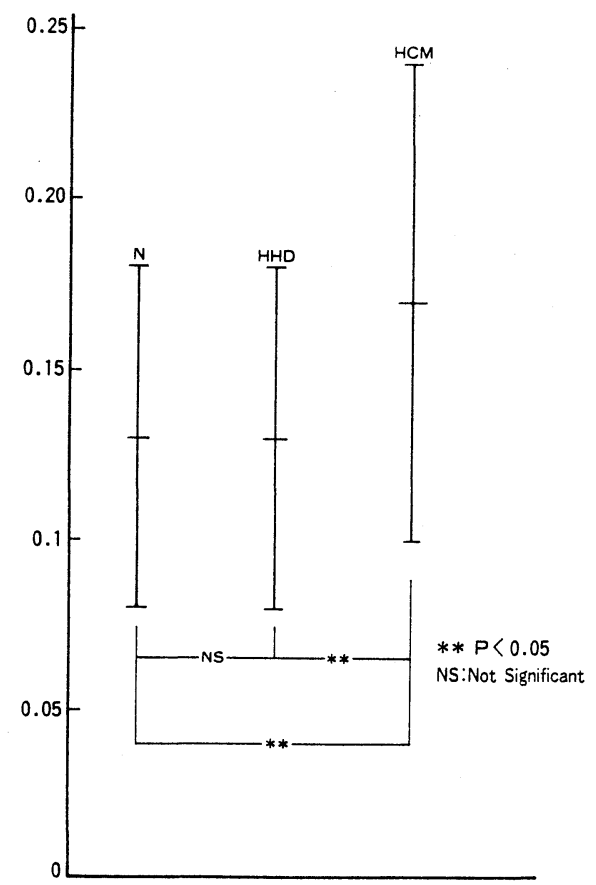

Fig. 5. The $\mathrm{c} / \mathrm{a}$ ratio of the spatial $\mathrm{QRS}$ loop. The ratio is significantly greater in the HCM group than in the other groups, whereas no significant difference is found between the HHD group and the normal group. Abbreviations are the same as Fig. 3.

\section{DISCUSSION}

When the coordinate axis is transformed to allow the spatial QRS loop to be observed from the front of a certain plane, the spatial characteristics of the loop can be clarified. In the present study, both the HHD and HCM groups, which had similar heart electrical forces recorded through the body surface, showed high values in the area and thickness of the spatial QRS loop, and no significant difference was found between them. In contrast, the thickness/length ratio in the HHD group was similar to that of the normal group, and the HCM group showed significantly greater values than the HHD group. The spatial QRS loop is reported to have planarity $(10,11)$; indicators of planarity have been reported by Okamoto and Simonson (12) and Yamauchi (13). Draper et al (14) defined the thickness/length ratio obtained by the method of least squares as an indicator of planarity. The thickness/length ratio was then calculated using the

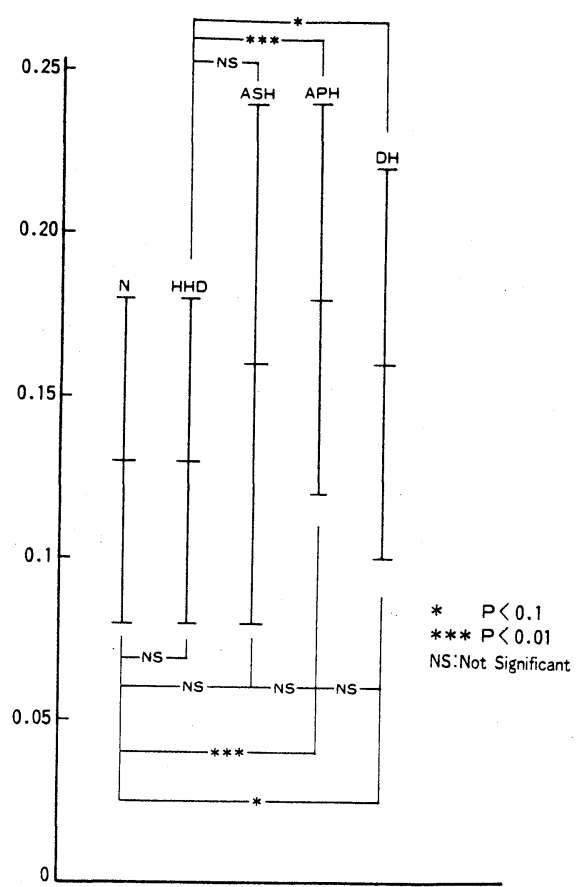

Fig. 6. The comparison of the $\mathrm{c} / \mathrm{a}$ ratio between HCM subgroups. There is no significant difference between the subgroups. Abbreviations are the same as Fig. 3.

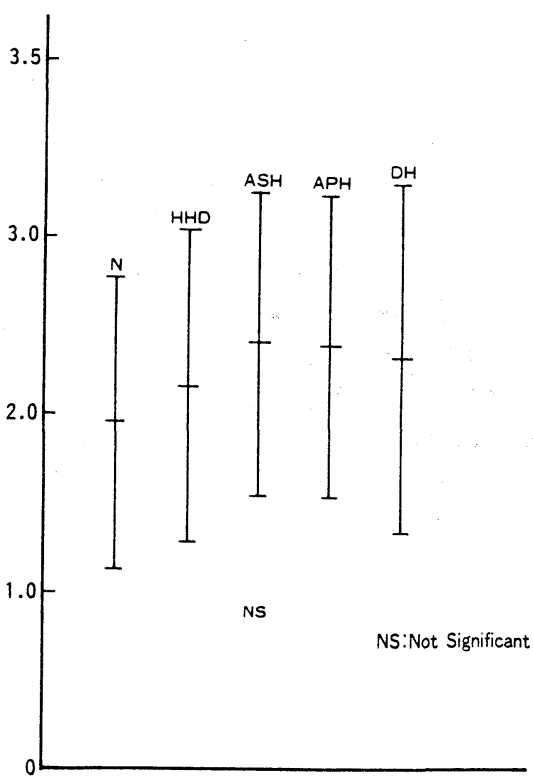

Fig. 7. The $a / b$ ratio of the QRS loop. There is no significant difference between the groups. Abbreviations are the same as Fig. 3. 
resolver method. This was used as an indicator of planarity in the present study. The results showed a greater decrease in planarity in the HCM group than in the HHD group, in other words, a greater deformation in the QRS loop.

VCG or most HCM patients shows no apparent bundle branch block patterns, but presents nonspecific intraventricular conduction disturbances such as QRS loop irregularity as a whole, irregular nodular formation, prolonged duration of the QRS loop and delayed inscription. Coyne (15) termed the QRS loop deformation in HCM the "pigtail curl," reporting that it is a finding specific to $\mathrm{HCM}$, and particularly to HOCM. However, this is a qualitative finding that lacks objectivity. In the present study, characteristic changes in the QRS loop, which have not been determined on conventional VCG, were clarified by quantitatively analyzing the deformation of the QRS loop.

One of the possible causes of this deformation involves histological changes of the myocardium in HCM. Burch and De Pasquale (16) reported that the disappearance of septal Q in HCM is caused by fibrosis of the ventricular septum, and Coyne (15) reported that complication with myocardial fibrosis or muscle fiber disarray may induce complicated stimulus conduction, resulting in diversity of ECG findings. The bizarre arrangement of myocardial cells that is specific to HCM may cause unequal orientation of each instantaneous vector of the heart electrical force, leading to apparent deformation resembling intraventricular conduction disturbance. Therefore, in the HHD group with left ventricular hypertrophy due simply to increased workload of the left ventricle, the planarity of the QRS loop is possibly maintained to some extent while the area or thickness is increased, creating a significant difference from the HCM group.

Since there have been reports that patients with severe hypertrophy do not necessarily show increased thickness/length ratio, there may be some relationship between the planarity of the QRS loop and the intraventricular conduction disturbance in HCM. In the present study, however, a comparison of histological changes in the whole myocardium was difficult, because myocardial biopsy was performed on a limited part of the right ventricular endocardium, and the relationship was not confirmed histopathologically. Moreover, since no patient in the HHD group had received myocardial biopsy, such a study was not possible. Carter and Estes (17) reported a close relationship between 12-lead ECG findings of left ventricular hypertrophy and autopsy findings of hypertrophy of the left ventricular wall and the weight of the left ventricular myocardium. Yamori et al (18) also reported the presence of VCG findings of left ventricular hypertrophy, significantly thickened myocardium, and increased weight of the left ventricle in spontaneously hypertensive rats (SHR), suggesting a close relationship between electrocardiographic changes and pathological changes of the myocardium. Therefore, it is highly likely that the differences in histological changes of the myocardium are reflected on electrocardiograms. We are presently investigating whether there is a correlation between the histopathological severity of HCM and the degree of "deformation in the spatial loop". It would be interesting from the viewpoint of electrocardiography, if the conclusion is that the histopathological difference is reflected clearly on the VCG.

Since HHD patients frequently show symmetrical ventricular wall thickening on echocardiograms, clinical differentiation from HCM, especially in the DH type, is often necessary. The significant difference in the planarity of the QRS loop on VCG between the two groups, as revealed by the present study, may provide a useful indicator for their differential diagnosis.

\section{REFERENCES}

1) Hollister RM, Goodwin JF. The electrocardiogram in cardiomyopathy. Br Heart J 25: 357, 1963.

2) Estes E, Whalen E, McIntosch DD. The electrocardiographic and vectorcardiographic findings in idiopathic hypertrophic subaortic stenosis. Am Heart J 65: 155, 1963.

3) Braunwald E, Lambrew CT, Rockoff SD, Ross J, Marrow AG. Idiopathic hypertrophic subaortic stenosis. I. A description of the disease based upon an analysis of 64 patients. Circulation 30 (Suppl. IV): 3, 1964.

4) Marriot $\mathrm{HJ}$. Electrocardiographic abnormalities, conduction disorders and arrhythmias in primary myocardial disease. Prog Cardiovasc Dis 7: 99, 1964.

5) Hamby RI, Rial F. Electrocardiographic aspects of primary myocardial disease in 60 patients. Am Heart J 76: 316,1968 .

6) Horan LG, Flowers NC, Thomas JR, Tolleson WJ. The spatial vectorcardiogram in idiopathic cardiomyopathy. 
Prog Cardiovasc Dis 7: 115, 1964.

7) Pipberger HV, Wood CR Jr. A simplified method of the resolution of the orthogonal electrocardiogram. Circ Res 6: 239, 1985.

8) Schmitt $\mathrm{OH}$. Cathode-ray presentation of threedimensional data. J Appl Physiol 18: 819, 1947.

9) Milnor WR, Talbot SA, Newman EV. A study of the relationship between unipolar leads and spatial vectorcardiograms, using the panoramic vectorcardiograph. Circulation 7: 545, 1953.

10) Pipberger HV, Carter TN. Analysis of the normal and abnormal vectorcardiogram in its own reference frame. Circulation 25: 827, 1962.

11) Ohshita K. Study on vectorcardiogram; Planarity of normal spatial vectorcardiogram. Jpn Circ J 30: 237, 1966.

12) Okamoto N, Simonson E. Separation of normal and abnormal vectorcardiograms. Use of spatial orientation of initial, central and terminal segmental planes of the QRS loop. Am J Cardiol 18: 682, 1966.

13) Yamauchi K. Computer analysis of vectorcardiograms in myocardial infarction with special reference to polar vector and planarity of the QRS and T loops. Jpn Heart J 20: 587, 1979.

14) Draper HW, Peffer CJ, Stallmann FW, Littmann D, Pipberger HV. The corrected orthogonal electrocardiogram and vectorcardiogram in 510 normal men. Circulation 30: 853, 1964.

15) Coyne JJ. New concepts of intramural myocardial conduction in hypertrophic obstructive cardiomyopathy. Br Heart J 30: 546, 1968.

16) Burch GE, De Pasquale N. A study at autopsy of the relation of absence of the $Q$ wave in lead $I, a V L, V_{5}$ and $V_{6}$ to septal fibrosis. Am Heart J 60: 336, 1960.

17) Carter WA, Estes EH Jr. Electrocardiographic manifestations of ventricular hypertrophy; A computer study of ECG-anatomic correlations in 319 cases. Am Heart J 68: 173, 1964.

18) Yamori $Y$, Ohtaka M, Nara Y. Vectorcardiographic study on left ventricular hypertrophy in spontaneously hypertensive rats. Jpn Circ J 40: 1315, 1976. 